material in the nuclei seems to be totally or partly due to the nuclear membrane and to the nucleoli, if present.

As suggested by ACKERMAN ${ }^{4}$, it might be assumed that lipids are present in the nuclei as lipoproteins or as a liponucleoprotein complex. As most lipids are presumably conjugated to proteins, only a highly sensitive reagent, such as benzpyrene, is able to demonstrate directly the few reagent hydrophobic radicals. Nuclear lipids become sudanophilic only after denaturation, at least partial, of the protein layer. As for the nuclear sudanophilia observed after treatment with fat solvents, an alternative hypothesis is to assume, that a 'sudanophobic' lipid coating (cholesterol?) must be removed before the sudano- philic lipid layer of the nuclear membrane becomes accessible to the dye.

Zusammenfassung. Die Anwesenheit von Lipiden im Kern der Zellen des Blutes und der hämopoetischen Organe wurde histochemisch nachgewiesen. Die Lipide scheinen ausschliesslich oder stark überwiegend in der Kernmembran und in den Nukleolen lokalisiert zu sein.

C. Mauri and V. Sillingakdi

Istituto di Patologia Medica, Università di Modena (Italy), May 13, 1963.

\section{Metallionen-katalysierte Hydrolyse von Adenosintriphosphat}

Der vor kurzem veröffentlichte Bericht von TETAs und LowensteIN ${ }^{1}$ iber die Katalyse der nichtenzymatischen Adenosintriphosphat (ATP)-Hydrolyse durch zweiwertige Metallionen veranlasst uns, über Untersuchungen ähnlicher Art zu berichten, die wir in Verfolgung unserer früheren Beobachtungen ${ }^{2,3}$ durchgeführt haben.

Durch chromatographische Untersuchungen auf Diäthylaminoäthyl (DEAE)-Zellulose konnten wir zunächst leicht feststellen, dass die früher erwähnte ${ }^{2,3}$ Instabilität des Komplexes [Cu-ATP] ${ }^{2-}$ im wesentlichen durch dessen Hydrolyse zu [Cu-ADP $]^{-}$und anorganischem Phosphat bedingt ist. Eine weitergehende Hydrolyse findet daneben nur in untergeordnetem Masse statt.

Die Kinetik der Hydrolysenreaktion verfolgten wir durch Messung der Phosphatfreisetzung 4 . Die Hydrolyse des ATP wird bei $50^{\circ}$ durch einen $\mathrm{Zusatz}$ von $\mathrm{Cu}^{2+}$-Ionen um einen Faktor von etwa 100 beschleunigt. Das Ausmass der $\mathrm{Cu}^{2+}$-Katalyse hängt stark vom $\mathrm{pH}$-Wert der Lösung $\mathrm{ab}$. Wir fanden, wie die genannten Autoren, bei pH 5,5-6 ein Maximum der Reaktionsgeschwindigkeit. Der Komplex $[\mathrm{Cu}-\mathrm{ATP}]^{2-}$, welcher bei diesem $\mathrm{pH}$-Wert in der Lösung vorliegt, kann einerseits ein Proton mit einem pK-Wert von ca. 4 anlagern ${ }^{3,5}$, anderseits bei höherem $\mathrm{pH}$ ein Proton abspalten ${ }^{2,5}$. Hierdurch wird die auch von TETAs und LowENSTEIN geäusserte Vermutung gestützt, dass die Ausbildung von $[\mathrm{Cu}-\mathrm{ATP}-\mathrm{H}]^{-} \mathrm{bzw}$. von $[\mathrm{Cu}-\mathrm{ATP}-\mathrm{OH}]^{3-}$ aus $[\mathrm{Cu}-\mathrm{ATP}]^{2-}$ die starke Verringerung der Hydrolysengeschwindigkeit $z u$ beiden Seiten des pH-Optimums verursacht. Wir können mithin den Befund bestätigen, dass für die beschleunigte Hydrolyse ein Komplex verantwortlich ist, dessen Protonierungsgrad tatsächlich der Formulierung $[\mathrm{Cu}-\mathrm{ATP}]^{2-}$ entspricht.

Dieser Befund reicht jedoch für eine sinnvolle Diskussion der katalytisch aktiven Komplexstrukturen und der zugehörigen Reaktionsmechanismen nicht aus, denn in der wässrigen Lösung eines Komplexes dieser stöchiometrischen Zusammensetzung treten eine Vielzahl strukturisomerer Komplexpartikel auf ${ }^{*}$. Um zu entscheiden, welche der vorkommenden Komplexkonformationen die hydrolytische Spaltung der Polyphosphatkette induziert, haben wir eine Reihe weiterer Experimente unternommen, als deren vorläufige Ergebnisse genannt seien: (1) Die Hydrolysenreaktion ist nicht erster Ordnung in bezug auf [Cu-ATP] ${ }^{2-}$, sondern zeigt eine starke Konzentrationsabhängigkeit; d.h. in einer Lösung dieses Komplexes muss die reaktive Partikel in einem konzentra- tionsabhängigen Gleichgewicht mit unreaktiven Partikeln stehen. (2) Hierauf weist auch das Ergebnis einer Untersuchung nach der JoBschen "Methode der kontinuierlichen Variation" hin: Die Hydrolysengeschwindigkeit zeigt ein Maximum beim Verhältnis $\mathrm{Cu}$ : ATP 1,2. (3) Gleichzeitig mit der Hydrolysenreaktion findet im $\mathrm{Nu}-$ kleotidkomplex, wie die völlige Umkehr der Rotationsdispersion anzeigt, offenbar eine drastische Konformationsumwandlung statt. (4) Der $\mathrm{Cu}^{2+-K o m p l e x}$ von Methyltriphosphat ( $\left.\mathrm{Cu}-\mathrm{MTP}]^{2-}\right)$ hydrolysiert ca. $60 \mathrm{mal}$ langsamer als $[\mathrm{Cu}-\mathrm{ATP}]^{2-}$, obwohl in beiden Komplexen IR-spektrophotometrisch die gleichen Bindungsverhältnisse zwischen Metallion und Polyphosphatkette zu beobachten sind ${ }^{6}$

Eine ausführliche Darstellung und Interpretation unserer Ergebnisse, deren weitere Verfolgung einen Ausblick auf die Mechanismen der dieser "Modellreaktion" entsprechenden enzymatischen Reaktionen erhoffen lässt, soll demnächst veröffentlicht werden ${ }^{7}$.

Summary. Some kinetic and structural data on the catalysis exerted by $\mathrm{Cu}^{2+}$ in the hydrolysis of ATP are given.

P. SCHNEIDER,

H. BRintzinger und H. ERLENMEYER

Institut für Anorganische Chemie der Universität Basel (Schweiz), 9. September 1963.

1 M. Tetas und J. M. Lowenstein, Biochemistry 2,350 (1963)

2 H. Brintringer, Helv. chim. Acta \$4, 1199 (1961).

3 U. Hanoscmin und H. Brintzinger, Helv. chim. Acta 45,1037 (1962).

4 Phosphatbestimmungen nach A. A. Hirata und D. Appleman, Analyt. Chem. 31, 2097 (1959).

5 M. M. Taqui Khan und A. E. Marteli, J. phys. Chem, 66, 10 (1962).

6. Brintzincer, Biochim, biophys, Acta $z 7,943$ (1063).

* Herrn Dr. H. SeIlek danken wir für wertvolle Hinweise für die chromatographischen Lntersuchungen. Dem Schweizerischen Nationalfonds zur Förderung der wissenschaftlichen Forschung sci an dieser Stelle für die gewährte Unterstïtzung bestens gedankt. 